\title{
Pelvic Floor Symptom Related Distress in Chronic Constipation Correlates With a Diagnosis of Irritable Bowel Syndrome With Constipation and Constipation Severity but Not Pelvic Floor Dyssynergia
}

\author{
Prashant Singh, Yoonjin Seo, Sarah Ballou, Andrew Ludwig, William Hirsch, Vikram Rangan, Johanna Iturrino, Anthony Lembo, \\ and Judy W Nee* \\ Division of Gastroenterology, Beth Israel Deaconess Medical Center, Boston, USA
}

\section{Background/Aims}

Although symptoms related to the pelvic floor, such as pelvic organ prolapse (POP) and lower urinary tract symptoms (LUTS), are common in patients with chronic constipation (CC), its impact is not clear. Our aims were to investigate the following (1) compare pelvic floor symptom related dysfunction in irritable bowel syndrome with constipation (IBS-C) and functional constipation (FC), and

(2) symptom correlation with findings on anorectal manometry (ARM) and balloon expulsion test.

\section{Methods}

This was a retrospective analysis of patients with CC undergoing ARM. IBS-C and FC were diagnosed by Rome III criteria. Pelvic Floor Distress Inventory (PFDI-20) was used to measure pelvic floor symptom distress. Constipation Severity Scale was used to assess constipation severity.

\section{Results}

A total of 107 patients underwent ARM (64 FC, 43 IBS-C). The overall PFDI-20 score in IBS-C was higher compared with FC patients (118.0 vs 79.2, $P=0.001)$. In those with IBS-C, POP, LUTS, and colorectal symptoms subscales were all higher compared with FC patients $(P<0.05$ for each). On multivariable regression, IBS-C $(P=0.001)$ and higher constipation severity $(P=0.001)$ were both independently associated with higher PFDI scores. ARM parameters and abnormal balloon expulsion test did not correlate with PFDI scores.

\section{Conclusions}

Compared with FC patients, those with IBS-C have significantly higher distress from pelvic floor specific symptoms including POP and LUTS. Higher abdominal pain among IBS-C patients did not entirely explain these findings. A diagnosis of IBS-C and higher constipation severity correlated with PFDI-20 scores, but dyssynergia did not.

(J Neurogastroenterol Motil 2019;25:129-136)

Key Words

Constipation; Defecation; Lower urinary tract symptoms; Manometry; Pelvic floor

Received: December 13, 2017 Revised: April 4, 2018 Accepted: September 28, 2018

(.) This is an Open Access article distributed under the terms of the Creative Commons Attribution Non-Commercial License (http://creativecommons. org/licenses/by-nc/4.0) which permits unrestricted non-commercial use, distribution, and reproduction in any medium, provided the original work is properly cited.

*Correspondence: Judy W Nee, MD

Division of Gastroenterology, Department of Medicine, Beth Israel Deaconess Medical Center and Harvard Medical School, 330 Brookline Avenue, Boston, MA 02115-5400, USA

Tel: +1-617-667-2135, Fax: +1-617-667-5826, E-mail: jnee@bidmc.harvard.edu 


\section{Introduction}

Chronic constipation (CC) is a common disorder, affecting nearly $15-20 \%$ of the population worldwide. ${ }^{1-3} \mathrm{CC}$ is often subdivided into irritable bowel syndrome with constipation (IBS-C) and functional constipation (FC) per Rome IV criteria though these entities exist in a continuum. ${ }^{3}$ The main difference between the 2 clinical diagnoses is that abdominal pain and bloating are not predominant symptoms are not predominant symptoms in $\mathrm{FC}^{3}{ }^{3} \mathrm{Up}$ to $50 \%$ of patients with $\mathrm{CC}$ are found to have defecatory disorder on further investigation. ${ }^{4}$ Patients with defecatory disorder have either (1) inadequate defecatory propulsion (defined by inadequate rectal propulsive forces as measured with anorectal manometry [ARM] with or without inappropriate contraction or incomplete relaxation of the anal sphincter and/or pelvic floor muscles) or (2) dyssynergic defecation (defined by paradoxical contraction or inappropriate relaxation of the pelvic floor as measured with anal surface electromyography or ARM with adequate propulsive forces during attempted defecation).,

Given the anatomic and functional role of pelvic floor in defecation, it is not surprising that constipation is often comorbid with other pelvic floor issues such as urinary symptoms and pelvic organ prolapse. ${ }^{6-11}$ Studies have shown that lower urinary tract symptoms such as urinary urgency, frequency, retention, and incontinence are more common in women with constipation compared to healthy controls. ${ }^{6,7}$ In addition, constipation has been showed to be significantly associated with pelvic organ prolapse in epidemiological as well as case-control studies. ${ }^{8-11}$

Despite the high prevalence of pelvic organ prolapse and lower urinary tract symptoms in individuals with constipation, little is known about their impact of these symptoms on $\mathrm{CC}$ patients. There are no studies investigating the predictors of pelvic floor symptom dysfunction in individuals with $\mathrm{CC}$ and it is unclear if this significantly varies between patients with IBS-C and FC. In this study, we aim to investigate if (1) patient reported pelvic floor symptom dysfunction measured by Pelvic Floor Distress Inventory (PFDI20 ) is significantly different among constipation subtypes (IBS-C vs FC), and (2) pelvic floor symptom dysfunction correlates with findings on ARM and balloon expulsion test (BET).

\section{Materials and Methods}

\section{Subjects}

All female patients undergoing ARM from December 2012 to June 2016 were consecutively enrolled at Beth Israel Deaconess Medical Center's (BIDMC) gastrointestinal motility laboratory for ARM. All the patients were asked to complete the Rome III questionnaire and the PFDI-20 as part of their clinical care. The institutional review board of BIDMC approved waiver of informed consent for this retrospective review. Individuals who met the Rome III criteria for IBS-C and FC were included in the study. Patients greater than 18 years in age were included in the study. Exclusion criteria included major anorectal or colonic surgery. Demographic details and clinical data were obtained through questionnaires. In addition, data from high-resolution ARM and BET were recorded.

\section{Three-dimensional High-resolution Manometry}

Anal pressures were assessed using the 3-dimensional highresolution manometry (3D-HRM) probe. The details of this probe is described in our previous study. ${ }^{12}$ Prior to the study, patients were instructed to fast for 4 hours and use 2 enemas. During ARM, patients lay in the left lateral decubitus position. After inserting the $3 \mathrm{D}-\mathrm{HRM}$ probe, the assessment of anorectal pressures was performed at rest, during 3 attempts of squeeze, and in 3 attempts of simulated defecation. Afterward, the recto-anal inhibitory anal reflex (RAIR) and rectal sensation thresholds were measured through progressive inflation of the intrarectal balloon with 10-20 $\mathrm{mL}$ increments. Balloon volumes at which the patient reported the first sensation, desire to defecate and severe urgency were noted. If the patient did not attain these sensations or RAIR until a balloon volume of $250 \mathrm{~mL}$, this was documented.

\section{Three-dimensional High-resolution Manometry Analysis}

Adequate rectal propulsion was defined as an intrarectal pressure of $>40 \mathrm{mmHg}$ during at least 1 of the simulated defecations. ${ }^{12}$ Adequate anal relaxation was defined as $>20 \%$ relaxation during at least 1 of the simulated defecations. ${ }^{13}$

\section{Balloon Expulsion Test}

BET was performed following 3D-HRM. With the patient in the left lateral decubitus, a non-latex $400 \mathrm{~mL}$ volume balloon was inserted into the rectum and inflated with $50 \mathrm{~mL}$ of warm water. 
The patient was then asked to sit on a commode in privacy and expel the balloon. BET was considered abnormal if the time required to expel the balloon was more than 1 minute. ${ }^{14}$

\section{Study Questionnaires}

Patients completed the English version of the PFDI-20 questionnaire and the Rome III Constipation Module prior to undergoing 3D-HRM. ${ }^{15,16}$ The PFDI measures symptom severity and evaluates patient's distress in pelvic floor disorders including pelvic organ prolapse, urinary incontinence, and colorectal dysfunction. The PFDI-20 has a total of 20 items and 3 subscales: 6 questions evaluating Urinary Distress Inventory (UDI-6), 6 questions evaluating Pelvic Organ Prolapse Distress Inventory (POPDI-6), and 8 questions evaluating Colorectal-Anal Distress Inventory (CRADI-8). ${ }^{15}$ If a symptom is present, it is scored from not at all (least distress) to quite a bit (greatest distress). PFDI-20 is a validated measure to assess pelvic floor symptom related dysfunction and has been in use for several years. ${ }^{15}$ Some studies have grouped the level of pelvic floor distress into mild, moderate, and severe based on total PFDI-20 score. ${ }^{15}$ Pelvic floor distress was considered mild if the total PFDI score was $\leq 100$, moderate if PFDI score was $>100$ and $\leq 200$ and severe if PFDI score was $>200 .^{17}$

The Rome III Constipation Module was used to classify patients as having FC or IBS-C. ${ }^{16}$ We used the Rome III questionnaire to assess the frequency of the following symptoms: (1) hard or lumpy stools, (2) straining during bowel movement, (3) feeling of incomplete evacuation after bowel movement, (4) sensation that stool is blocked when having a bowel movement, (5) need for digitalization to complete a bowel movement, and (6) $<3$ bowel movements per week. For each of these symptoms patients who reported these symptoms as "most of the time" or "always" were considered to have them frequently.

In addition, the Constipation Severity Scale (CSS) a previously validated measure of constipation severity was generated using the sum of the 5 -point ordinal ratings $(0=$ never, $1=$ sometimes, $2=$ often, $3=$ most of the time, and $4=$ always) for the 6 Rome III FC questions mentioned above. ${ }^{18}$

\section{Statistical Methods}

Continuous variables were summarized using means and $95 \%$ confidence intervals (CI) for normally distributed data. For continuous variables which were not normally distributed, medians and interquartile range were calculated. Baseline demographic, clinical, and ARM variables were compared between patients with IBS$\mathrm{C}$ and FC. POPDI-6, CRADI-8, UDI-6, and PFDI-20 scores were generated using the PFDI-20 scoring system. The mean value of all answered items within the corresponding scale (possible values $0-4$ ) were obtained and multiplied by 25 to generate the scale score (range 0 to100). Missing items are dealt with by using the mean from answered items only. Mean scores of POPDI-6, CRADI-8 and UDI-6 as well as overall mean PFDI-20 scores were compared between IBS-C and FC.

Proportions were compared using chi square test and means and medians were compared using Student's $t$ test and Wilcoxon rank-sum test respectively. Multivariable linear regression was used to determine the association among PFDI-20 score and demographics, 3D-HRM and BET parameters, constipation phenotype and clinical characteristics. Two questions pertaining to incomplete evacuation of stool and straining with bowel movement are present in both CSS and PFDI-20. Therefore, for the purpose of multivariable regression, these 2 questions were excluded from the CSS, and constipation severity in multivariable regression was assessed using the remaining 4 questions included in CSS. For all statistical analyses, $P$-value $<0.05$ was considered statistically significant.

\section{Results}

\section{Demographic Characteristics}

During the study period, 64 patients met the Rome III criteria for FC and 43 patients for IBS-C. The mean age of patients with FC was significantly higher than that of IBS-C (Table 1).

\section{Clinical Symptoms}

Proportions of patients with frequent straining, feeling of incomplete evacuation, sensation of blocked stool during bowel movement, need for digitalization to complete a bowel movement, hard or lumpy stools and $<3$ bowel movements/week were similar in IBS-C and FC groups (Table 1).

\section{Three-dimensional High-resolution Manometry}

The mean resting anal pressure, mean resting rectal pressure and mean squeeze pressure were comparable among patients with IBS-C and FC (Table 2). The median volume required to elicit RAIR was not significantly different between the 2 groups. The mean volume required to generate the first sensation, desire to defecate and severe urgency were also similar between the patients with IBS-C and FC. Proportions of patients with adequate rectal propulsion on simulated defecation were comparable between the 2 groups $(25.4 \%$ in FC and $27.5 \%$ in IBS-C, $P=0.818)$. About 
Table 1. Demographic and Clinical Characteristics of Patients With Functional Constipation and Irritable Bowel Syndrome With Constipation

\begin{tabular}{|c|c|c|c|}
\hline Demographic and clinical characteristics & $\mathrm{FC}(\mathrm{n}=64)$ & IBS-C $(n=43)$ & $P$-value \\
\hline Mean age (yr) & $50(46-53)$ & $41(37-46)$ & 0.003 \\
\hline Proportion of patients with frequent hard or lumpy stools & $34 / 63(54.0)$ & $28 / 42(66.7)$ & 0.20 \\
\hline Proportion of patients with 0-2 bowel movements/wk most of the time or always & $31 / 62(50.0)$ & $23 / 43(53.5)$ & 0.725 \\
\hline Proportion of patients with frequent straining & $49 / 64(76.6)$ & $30 / 41(73.2)$ & 0.694 \\
\hline Proportion of patients with frequent incomplete evacuation & $42 / 63(66.7)$ & $30 / 41(73.2)$ & 0.482 \\
\hline Proportion of patients with frequent feeling of blocked stool when having bowel movement & $37 / 64(57.8)$ & $27 / 41(65.9)$ & 0.410 \\
\hline Proportion of patients with frequent digitalization & $18 / 63(28.6)$ & $12 / 41(29.3)$ & 0.939 \\
\hline Constipation severity scale & $14.5(13.2-15.9)$ & $16.1(14.5-17.7)$ & 0.151 \\
\hline
\end{tabular}

FC, functional constipation; IBS-C, irritable bowel syndrome with constipation.

Values are expressed as mean $(95 \% \mathrm{CI})$ or $\mathrm{n}(\%)$.

Table 2. Comparison of Anorectal Manometry Characteristics of Patients With Functional Constipation and Irritable Bowel Syndrome With Constipation

\begin{tabular}{|c|c|c|c|}
\hline Anorectal manometry characteristics & $\mathrm{FC}(\mathrm{n}=64)$ & IBS-C $(n=43)$ & $P$-value \\
\hline Resting anal pressure (mean $[95 \% \mathrm{CI}], \mathrm{mmHg}$ ) & $70.6(62.9-78.4)$ & $75.0(66.5-83.6)$ & 0.455 \\
\hline Squeeze pressure (mean $[95 \% \mathrm{CI}], \mathrm{mmHg})$ & $176.4(161-191.9)$ & $177.5(154.9-200.1)$ & 0.934 \\
\hline Resting rectal pressure (mean [95\% CI], mmHg) & $89.2(78.4-100.2)$ & $86.9(68.4-105.4)$ & 0.813 \\
\hline Volume of first sensation (mean $[95 \% \mathrm{CI}], \mathrm{mL}$ ) & $29.8(25.7-34.0)$ & $31.0(25.7-36.2)$ & 0.735 \\
\hline Volume for desire to defecate (mean $[95 \% \mathrm{CI}], \mathrm{mL}$ ) & $80.5(71.6-89.5)$ & $84.0(72.1-95.8)$ & 0.638 \\
\hline Volume for urgency (mean [95\% CI], mL) & $105.1(94.0-116.3)$ & $114.6(99.7-129.5)$ & 0.292 \\
\hline Volume needed for RAIR (median [IQR], mL) & $20(20-40)$ & $20(10-40)$ & 0.706 \\
\hline Defecatory index (median [IQR]) & $0.3(0.2-0.5)$ & $0.3(0.1-0.4)$ & 0.332 \\
\hline Highest rectal bear down pressure with defecation (median [IQR], mmHg) & $24.9(17.9-40.2)$ & $24.1(12.5-42.1)$ & 0.738 \\
\hline $\begin{array}{l}\text { Proportion of patients with adequate rectal propulsive force during simulated } \\
\text { defecation }(\mathrm{n}[\%])\end{array}$ & $15 / 59(25.4)$ & $11 / 40(27.5)$ & 0.818 \\
\hline Highest percent anal relaxation during simulated defecation (median [IQR], \%) & $5(-30-20)$ & $2.6(-10-16.5)$ & 0.980 \\
\hline $\begin{array}{l}\text { Proportion of patients with adequate percent anal relaxation during simulated } \\
\text { defecation }(\mathrm{n}[\%])\end{array}$ & $14 / 59(23.7)$ & $6 / 40(15.0)$ & 0.288 \\
\hline Patients with prolonged balloon expulsion (n [\%]) & $32 / 60(53.3)$ & $19 / 43(44.2)$ & 0.360 \\
\hline
\end{tabular}

FC, functional constipation; IBS-C, irritable bowel syndrome with constipation; RAIR, recto-anal inhibitory reflex; IQR, interquartile range.

$23.7 \%$ of the patients with FC and $15.0 \%$ of the patients with IBS-

$\mathrm{C}$ had adequate percentage anal relaxation on simulated defecation $(P=0.288)$.

\section{Balloon Expulsion Test}

Of 107 patients, 103 (60 with FC and 43 with IBS-C) underwent BET along with ARM. Of these, 32 patients with FC (53.3\%) and 19 patients with IBS-C (44.2\%) had prolonged BET based on $>1$ minute as upper limit of normal, which was not significantly different (Table 2).

\section{Pelvic Floor Distress Inventory-20 Score and Sub- scores by Constipation Subtype}

The mean POPDI-6 score was significantly higher among patients with IBS-C compared with FC (38.2 vs 25.0, $P=0.004$ ).
IBS patients also had higher CRADI-8 score compared with patients with $\mathrm{FC}$ (46.5 vs 37.6, $P=0.04$ ). The mean UDI-6 score was also higher among patients with IBS-C than patients with FC (33.7 vs $19.5, P=0.01$ ). The overall PFDI score was also significantly higher among patients with IBS-C in comparison with patients with FC (Table 3).

There was a significant difference in the severity of pelvic floor distress between patients with IBS-C and FC ( $P=0.007)$. IBS-C patients were more likely to have moderate (PFDI $>100$ and $\leq$ $200 ; 57.1 \%$ vs $26.5 \%$ ) or severe (PFDI score was $>200 ; 5.7 \%$ vs $2.0 \%$ ) compared to FC patients. Figure shows the comparison of the severity of pelvic floor distress among patients with IBS-C and FC. 
Table 3. Comparison of Pelvic Floor Distress Inventory and Sub-scores Between Patients With Functional Constipation and Irritable Bowel Syndrome With Constipation

\begin{tabular}{lccc}
\hline \multicolumn{1}{c}{ Pelvic Floor Distress Inventory scores } & FC $(\mathrm{n}=64)$ & IBS-C (n $=43)$ & $P$-value \\
\hline Pelvic organ prolapse distress inventory score & $25.0(19.4-30.6)$ & $38.2(31.0-45.4)$ & 0.004 \\
Colorectal anal distress inventory score & $37.6(32.0-43.3)$ & $46.5(39.6-53.3)$ & 0.041 \\
Urinary distress inventory score & $19.5(12.7-26.2)$ & $33.7(24.9-42.5)$ \\
Pelvic floor distress inventory score & $79.2(64.9-93.6)$ & $118.0(99.6-136.3)$ & 0.010 \\
\hline
\end{tabular}

FC, functional constipation; IBS-C, irritable bowel syndrome with constipation.

Values are expressed as mean $(95 \% \mathrm{CI})$.

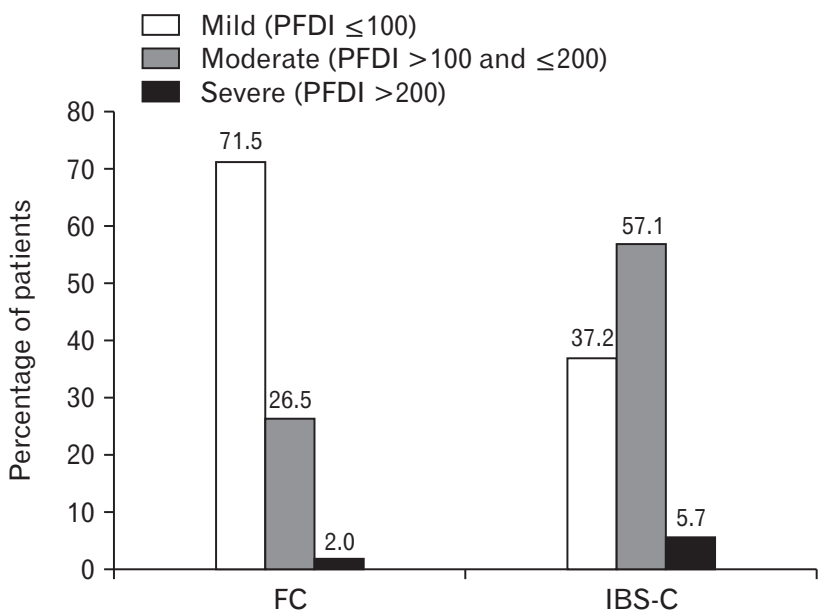

Figure. Comparing the severity of pelvic floor distress among patients with functional constipation (FC) and irritable bowel syndrome with constipation (IBS-C). IBS-C patients were more likely to have moderate or severe Pelvic Floor Distress Inventory (PFDI) score compared to $\mathrm{FC}$ patients.

\section{Individual Pelvic Floor Distress Inventory-20 Question by Constipation Subtype}

To understand the symptoms underlying the higher distress among the IBS-C patients, we compared individual PFDI-20 question between the 2 groups. IBS-C patients had higher mean scores compared with FC patients when asked about (1) pressure in lower abdomen, (2) heaviness or dullness in lower abdomen, and (3) pain or discomfort in lower abdomen or genital region (Table 4). Patients with IBS-C also reported higher mean distress than FC patients about the following symptoms - feeling of incomplete bladder emptying, feeling of incomplete bowel evacuation at the end of a bowel movement, stool bulging out of rectum during or after bowel movement, pain while passing stools, frequent urination, and difficulty in emptying bladder (Table 4). There was no significant difference between the 2 groups when the remaining individual PFDI-20 questions were compared between the 2 groups.

\section{Multivariable Regression}

On multivariable regression, diagnosis of IBS-C (coefficient of regression, 41.6; 95\% CI, 16.6-66.6; $P=0.001$ ) and higher constipation severity (coefficient of regression, 5.3; 95\% CI, 2.4-8.3; $P=0.001)$ were independently associated with higher PFDI scores (Table 5).

In a subset of patients in whom detailed obstetric history was available (77 patients), 23 patients (29.9\%) were nulliparous. Of the remaining 54 women who had children, 39 women $(72.2 \%)$ had only vaginal deliveries. Thirteen of these 77 patients (16.9\%) had a prior history of hysterectomy. We tried identifying independent predictors of PFDI-20 in this subset of patients including the variables of nulliparity, history of hysterectomy, and only vaginal deliveries in addition to other variables included in Table 5. While IBS-C and higher constipation severity continued to be independently associated with higher PFDI scores, history of only vaginal deliveries was also independently associated with higher PFDI scores (coefficient of regression, $41.3 ; 95 \% \mathrm{CI}, 4.5-78.2 ; P=0.030)$.

\section{Discussion}

In this study, we found that the mean PFDI-20 score (and its sub-score) did not have any significant correlation with prolonged BET and/or 3-D HRM variables. The mean PFDI-20 score, which describes patient's distress due to the myriad of pelvic floor symptoms, (and its sub-scores) were significantly higher among patients with IBS-C compared with patients with FC. Analysis of the individual PFDI-20 question suggested that the higher pelvic floor distress scores among patients with IBS-C was not entirely due to higher pain scores in the lower abdominal/pelvic area experienced by these patients. Ours is the first study showing that overall pelvic floor symptom dysfunction in patients with $\mathrm{CC}$ correlates with diagnosis of IBS-C and constipation severity.

Although several studies have shown association between pelvic floor symptoms and CC, studies exploring pelvic floor dyssynergia 
Table 4. Comparison of Individual Pelvic Floor Distress Inventory Questions Between Patients With Functional Constipation and Irritable Bowel Syndrome With Constipation

\begin{tabular}{|c|c|c|c|}
\hline Individual PFDI-20 question ${ }^{a}$ & $\mathrm{FC}(\mathrm{n}=64)$ & IBS-C $(n=43)$ & $P$-value \\
\hline Do you usually experience pressure in lower abdomen? & $1.5(1.1-1.9)$ & $2.5(2.1-3.0)$ & 0.002 \\
\hline Do you usually experience dullness or heaviness in the lower abdomen? & $1.5(1.0-1.9)$ & $2.4(1.9-2.8)$ & 0.006 \\
\hline Do you usually experience a feeling of incomplete bladder emptying? & $1.1(0.7-1.4)$ & $1.7(1.2-2.2)$ & 0.030 \\
\hline $\begin{array}{l}\text { Do you feel you have not completely emptied your bowels at the end of a bowel } \\
\text { movement? }\end{array}$ & $3.1(2.7-3.4)$ & $3.6(3.3-3.8)$ & 0.043 \\
\hline Do you usually have pain when you pass your stool? & $1.6(1.1-2.1)$ & $2.6(2.1-3.1)$ & 0.005 \\
\hline $\begin{array}{l}\text { Does part of your stool ever pass through the rectum and bulge outside during or } \\
\text { after a bowel movement? }\end{array}$ & $0.9(0.5-1.4)$ & $1.6(1.0-2.3)$ & 0.049 \\
\hline Do you usually experience frequent urination & $1.1(0.6-1.5)$ & $1.8(1.2-2.4)$ & 0.046 \\
\hline Do you usually experience difficulty emptying your bladder? & $0.3(0.1-0.5)$ & $0.9(0.3-1.4)$ & 0.028 \\
\hline Do you usually experience pain or discomfort in the lower abdomen or genital region? & $0.9(0.5-1.3)$ & $1.7(1.1-2.3)$ & 0.026 \\
\hline
\end{tabular}

anly questions with statistical significance $(P$-value $<0.05)$ are shown in this table.

PFDI, pelvic floor distress inventory; FC, functional constipation; IBS-C, irritable bowel syndrome with constipation.

Values are expressed as mean $(95 \% \mathrm{CI})$.

Table 5. Multivariable Regression to Determine Variables Correlating With Overall Pelvic Floor Distress Inventory Score

\begin{tabular}{lcc}
\multicolumn{1}{c}{ Independent variables } & Coefficient of regression (95\% CI) & $P$-value \\
\hline Age (yr) & $0.2(-0.7-1.1)$ & 0.619 \\
IBS-C & $41.6(16.6-66.6)$ & 0.001 \\
Constipation severity & $5.3(2.4-8.3)$ & 0.001 \\
Presence of prolonged balloon expulsion test time & $-4.9(-28.4-18.6)$ & 0.679 \\
Presence of adequate rectal propulsive force on simulated defecation & $2.8(-26.2-31.7)$ & 0.849 \\
Presence of adequate percent anal relaxation on simulated defecation & $12.9(-18.2-44.1)$ & 0.410 \\
\hline
\end{tabular}

IBS-C, irritable bowel syndrome with constipation.

as the underlying factor of this association are lacking. Ours is the first study to evaluate the correlation between pelvic floor symptom related distress and objective findings of pelvic floor dyssynergia in patients with $\mathrm{CC}$. We did not find any association between abnormal BET or abnormal 3D-HRM findings and higher PFDI scores. In a study by Chou et al, patients with severe obstructive defecation symptoms had worse PFDI-20 scores than patients with severe colonic inertia symptoms. ${ }^{19}$ However, this may have been affected by the significantly higher constipation severity symptoms in both groups. ${ }^{19}$ Furthermore, the prevalence of abnormal BET was similar in 2 groups. ${ }^{19}$ Thus, it is unclear that the association between PFDI-20 score and obstructive defecation seen in their study was truly due to pelvic floor dyssynergia or not. Our data suggests that PFDI scores independently correlates with severity of constipation and diagnosis of IBS-C.

While several studies have investigated the association of individual pelvic floor complaints such as pelvic organ prolapse, urinary symptoms, colorectal symptoms, etc and CC, ours is one of the few studies assessing the overall burden from pelvic floor related symptoms in these patients using a tool that provides global assessment of pelvic floor symptom dysfunction. ${ }^{19}$ Beyond suffering from symptoms associated with constipation, we found that the patients with FC and IBS-C have significant distress from pelvic organ prolapse as well as urinary symptoms. About $40 \%$ of our patient population had moderate to severe pelvic floor distress based on their PFDI20 scores. However, moderate to severe PFDI-20 scores were more common among IBS-C patients compared to FC patients. In fact, the mean score of PFDI-20 in patients with IBS-C was comparable to patients undergoing pelvic floor surgeries for pelvic floor symptoms in other studies. ${ }^{20,21}$ Indeed, a recent study has shown that for women with pelvic organ prolapse, a pre-operative PFDI-20 score $>98.45$ had $88 \%$ probability of significant improvement with prolapse surgery. $^{21}$

Patients with IBS-C also had higher distress from urinary symptoms compared with patients with FC. They reported higher distress from urinary frequency as well as difficulty in emptying the 
bladder. In a case-control study, Carter and Beer-Gabel ${ }^{6}$ did not show any significant difference in the frequency of lower urinary tract symptoms among patients with IBS-C and FC. However, they did not measure the level of distress from these symptoms in these patients. It is possible that underlying higher visceral hypersensitivity among IBS patients is not only restricted to the gut, but also involves other organ systems leading to higher distress from non-gastrointestinal symptoms. Irritability of the bladder and bowel occur in overactive bladder (defined by urinary urgency and urge incontinence) and IBS, respectively. In addition, many patients suffer from both conditions. ${ }^{22}$ Rodent experiments have supported the possibility of neural cross-talk between the two organ systems. Hyperesthesia of the bladder can be found in a colitis-associated colon-hyperalgesia model, whereas in a cyclophosphamide-induced cystitis model, colonic hypersensitivity has been noted. ${ }^{23,24}$

We found that patients with IBS-C have higher distress from pelvic organ prolapse by POPDI-6. This was largely due to questions related to distress from lower abdominal pressure, lower abdominal heaviness and pain while passing stool. Several studies have shown that overall abdominal pain reported among patients with IBS-C is higher than FC patients, and indeed abdominal pain is part of the diagnostic criteria of IBS-C and not $\mathrm{FC}^{25}$ It is possible that higher abdominal pain in IBS-C patients is not entirely related to the gastrointestinal tract and is in part due to other pelvic floor related symptoms such as pelvic organ prolapse, interstitial cystitis, etc.

Colorectal symptoms by PFDI-20 were also significantly worse in the IBS-C patients compared to FC. Although the frequency of symptoms of incomplete bowel evacuation after a bowel movement was similar among patients with IBS-C and FC, patients with IBS$\mathrm{C}$ reported higher distress from this symptom. Others have also shown that patients with IBS-C have higher severity of constipation specific symptoms, more distress from constipation symptoms and greater impairment in their quality of life when compared with patients with FC. ${ }^{25-27}$ Patients with IBS-C have also been shown to have higher underlying visceral hypersensitivity compared with FC patients as well as significantly higher rates of depression and anxiety. ${ }^{25,26}$ Several factors including higher levels of abdominal pain, higher severity of constipation, presence of visceral hypersensitivity and psychiatric comorbidities could explain higher distress from pelvic organ prolapse and colorectal-anal symptoms in these patients.

Our study has several limitations. First, ours is a cross sectional study and causation cannot be ascertained from this study. Second, the data is from a tertiary care center and does not necessarily repre- sent patients with constipation in the general population. Third, we lack some data related to the confounders that may affect anorectal function such as obstetric history in all patients, structural disease such as severity of pelvic organ prolapse, and psychiatric comorbidities. Similarly, diagnosis of perineal descent, rectal intussusception, etc could not be made as defecography was not performed in all the patients and future studies should investigate the burden on structural abnormalities on pelvic floor distress in patients with constipation.

In conclusion, this study showed that patients with constipation have significant distress from pelvic floor specific symptoms including urinary symptoms and pelvic organ prolapse. These findings were not entirely explained by higher abdominal pain or discomfort. Pelvic floor specific distress is associated with a diagnosis of IBS$\mathrm{C}$ (compared with FC) and higher constipation severity. It does not correlate with abnormal BET and/or 3-D HRM, suggesting that dyssynergia does not correlate with pelvic floor distress. Further studies should investigate the various factors underlying the higher distress related to pelvic floor symptoms among IBS-C patients compared to patients with FC.

Financial support: This project was funded in part by $\mathrm{Na}-$ tional Institutes of Health grants RO1AT008573-03 (A.L.) and 5T32DK007760-19 (P.S.).

\section{Conflicts of interest: None.}

Author contributions: Anthony Lembo and Judy W Nee planned and designed the research study; Yoonjin Seo, William Hirsch, and Andrew Ludwig contributed to data collection; Judy W Nee, Johanna I Moreda, and Anthony Lembo analyzed the manometric data; Prashant Singh conducted the statistical analysis; Prashant Singh, Judy W Nee, Anthony Lembo, Johanna I Moreda, Vikram Rangan, and Sarah Ballou drafted the manuscript; and all authors approved the final version of the article, including the authorship list.

\section{References}

1. Lembo A, Camilleri M. Chronic constipation. N Engl J Med 2003;349:1360-1368.

2. Sanchez MI, Bercik P. Epidemiology and burden of chronic constipation. Can J Gastroenterol J Can Gastroenterol 2011;25(suppl B):11B$15 \mathrm{~B}$.

3. Mearin F, Lacy BE, Chang L, et al. Bowel Disorders. Gastroenterology 2015;150:1393-1407, e5. 
4. Videlock EJ, Lembo A, Cremonini F. Diagnostic testing for dyssynergic defecation in chronic constipation: meta-analysis. Neurogastroenterol Motil 2013;25:509-520.

5. Schmulson MJ, Drossman DA. What is wew in Rome IV. J Neurogastroenterol Motil2017;23:151-163.

6. Carter D, Beer-Gabel M. Lower urinary tract symptoms in chronically constipated women. Int Urogynecology J 2012;23:1785-1789.

7. Wyndaele M, De Winter BY, Pelckmans PA, De Wachter S, Van Outryve M, Wyndaele JJ. Exploring associations between lower urinary tract symptoms (LUTS) and gastrointestinal (GI) problems in women: a study in women with urological and GI problems vs a control population. BJU Int 2015;115:958-967.

8. Akter F, Gartoulla P, Oldroyd J, Islam RM. Prevalence of, and risk factors for, symptomatic pelvic organ prolapse in Rural Bangladesh: a crosssectional survey study. Int Urogynecology J 2016;27:1753-1759.

9. Rortveit G, Subak LL, Thom DH, et al. Urinary incontinence, fecal incontinence and pelvic organ prolapse in a population-based, racially diverse cohort: prevalence and risk factors. Female Pelvic Med Reconstr Surg 2010;16:278-283.

10. Varma MG, Hart SL, Brown JS, Creasman JM, Van Den Eeden SK, Thom DH. Obstructive defecation in middle-aged women. Dig Dis Sci 2008;53:2702-2709.

11. Jelovsek JE, Walters MD, Paraiso MF, Barber MD. Functional bowel disorders and pelvic organ prolapse: a case-control study. Female Pelvic Med Reconstr Surg 2010;16:209-214.

12. Zakari M, Nee J, Hirsch W, Kuo B, Lembo A, Staller K. Gender differences in chronic constipation on anorectal motility. Neurogastroenterol Motil 2017;29.

13. Rao SSC, Patcharatrakul T. Diagnosis and treatment of dyssynergic defecation. JNeurogastroenterol Motil 2016;22:423-435.

14. Rao SS, Bharucha AE, Chiarioni G, et al. Functional anorectal disorders. Gastroenterology 2016;150:1430-1442.e4.

15. Barber MD, Kuchibhatla MN, Pieper CF, Bump RC. Psychometric evaluation of 2 comprehensive condition-specific quality of life instruments for women with pelvic floor disorders. Am J Obstet Gynecol 2001;185:1388-1395.

16. Shih DQ, Kwan LY. All roads lead to Rome: update on Rome III criteria and new treatment options. Gastroenterol Rep 2007;1:56-65.
17. Nosti PA, McDermott CD, Schilder JM, Stehman FB, Woodman PJ. Symptoms of pelvic floor disorders and quality of life measures in postoperative patients with endometrial cancer. Clin Ovarian Gynecol Cancer 2012;5:27-30.

18. Palsson OS, Turner MJ, Levy RL, et al. M1799 the Rome III questionnaire functional constipation module as a constipation severity scale. Gastroenterology 2008;134:A-421.

19. Chou AB, Cohan JN, Varma MG. Differences in symptom severity and quality of life in patients with obstructive defecation and colonic inertia. Dis Colon Rectum 2015;58:994-998.

20. Barber MD, Walters MD, Bump RC. Short forms of two conditionspecific quality-of-life questionnaires for women with pelvic floor disorders (PFDI-20 and PFIQ-7). Am J Obstet Gynecol 2005;193:103-113.

21. Laas E, Haddad M, Muhlstein J, Bendifallah S, Ballester M, Darai E. Preoperative quality of life questionnaires are an adequate tool to select women with genital prolapse for laparoscopic sacrocolpopexy. Int Urogynecology J 2017;28:1833-1839.

22. Matsumoto S, Hashizume K, Wada N, et al. Relationship between overactive bladder and irritable bowel syndrome: a large-scale internet survey in Japan using the overactive bladder symptom score and Rome III criteria. BJU Int 2013;111:647-652.

23. Qin C, Malykhina AP, Akbarali HI, Foreman RD. Cross-organ sensitization of lumbosacral spinal neurons receiving urinary bladder input in rats with inflamed colon. Gastroenterology 2005;129:1967-1978.

24. Bielefeldt K, Lamb K, Gebhart GF. Convergence of sensory pathways in the development of somatic and visceral hypersensitivity. Am J Physiol Gastrointest Liver Physiol 2006;291:G658-G665.

25. Shekhar C, Monaghan PJ, Morris J, et al. Rome III functional constipation and irritable bowel syndrome with constipation are similar disorders within a spectrum of sensitization, regulated by serotonin. Gastroenterology 2013;145:749-757.

26. Siah KT, Wong RK, Whitehead WE. Chronic constipation and constipation-predominant IBS: separate and distinct disorders or a spectrum of disease? Gastroenterol Hepatol 2016;12:171-178.

27. Wong RK, Palsson OS, Turner MJ, et al. Inability of the Rome III criteria to distinguish functional constipation from constipation-subtype irritable bowel syndrome. Am J Gastroenterol 2010;105:2228-2234. 